\title{
Dissipationless shock waves in Bose-Einstein condensates with repulsive interaction between atoms
}

\author{
A. M. Kamchatnov, ${ }^{1,2, *}$ A. Gammal, ${ }^{3, \dagger}$ and R. A. Kraenkel ${ }^{2,+}$ \\ ${ }^{1}$ Institute of Spectroscopy, Russian Academy of Sciences, Troitsk 142190, Moscow Region, Russia \\ ${ }^{2}$ Instituto de Física Teórica, Universidade Estadual Paúlista-UNESP, Rua Pamplona 145, 01405-900 São Paulo, Brazil \\ ${ }^{3}$ Instituto de Física, Universidade de São Paulo, 05315-970, C.P. 66318 São Paulo, Brazil
}

(Received 29 August 2003; published 10 June 2004)

\begin{abstract}
We consider formation of dissipationless shock waves in Bose-Einstein condensates with repulsive interaction between atoms. It is shown that for big enough initial inhomogeneity of density, interplay of nonlinear and dispersion effects leads to wave breaking phenomenon followed by generation of a train of dark solitons. Analytical theory is confirmed by numerical simulations.
\end{abstract}

DOI: 10.1103/PhysRevA.69.063605

PACS number(s): 03.75.Kk

Experiments on free expansion of Bose-Einstein condensate (BEC) have shown [1] that evolution of large and smooth distributions of BEC is described very well by hydrodynamic approximation [2] where dispersion and dissipation effects are neglected. At the same time, it is well known from classical compressible gas dynamics (see, e.g., [3]) that typical initial distributions of density and velocity can lead to wave breaking phenomenon when formal solution of hydrodynamical equations becomes multivalued. It means that near the wave breaking point one cannot neglect dispersion and/or dissipation effects which prevent formation of a multivalued region of a solution. If the dissipation effects dominate the dispersion ones, then the multivalued region is replaced by the classical shock, i.e., narrow layer with strong dissipation within, which separates smooth regions with different values of density, fluid velocity and other physical parameters. This situation was studied in classical gas dynamics and found many practical applications. If, however, the dispersion effects dominate dissipation ones, then the region of strong oscillations is generated in the vicinity of the wave breaking point $[4,5]$. Observation of dark solitons in BEC [6-8] shows that the main role in dynamics of BEC is played by dispersion and nonlinear effects taken into account by the standard Gross-Pitaevskii (GP) equation [9], and dissipation effects are relatively small and can be considered as perturbation. Hence, there are initial distributions of BEC which can lead to formation of dissipationless shock waves. Here we shall consider such a possibility.

The starting point of our consideration is the fact that the sound velocity in BEC is proportional to the square root from its density (see, e.g., [9] and references therein). Thus, if we create inhomogeneous BEC with high density hump (with density $\sim \rho_{1}$ ) in the center of lower density distribution (with density $\sim \rho_{0}$ ), and after that release this central part of BEC, then the high density hump will tend to expand with velocity $\sim \sqrt{\rho_{1}}$ greater than the sound velocity $\sim \sqrt{\rho_{0}}$ of propagation of disturbance in lower density BEC. As a result,

\footnotetext{
*Electronic address: kamch@isan.troitsk.ru

†Electronic address: gammal@if.usp.br

*Electronic address: kraenkel@ift.unesp.br
}

wave breaking and formation of dissipationless shock wave can occur in this case. Note that initial distributions of this type were realized in experiment [10] on measurement of sound velocity in BEC and in the recent experiment [11]. In [10] the hump's density $\rho_{1}$ was too small to generate shocks (see below). In experiment [11] generation of shock oscillations was apparently observed.

The theory of dissipationless shock waves in media described by a one-dimensional (1D) nonlinear Schrödinger (NLS) equation was developed in $[12,13]$. Since the GP equation in some cases can be reduced to the 1D NLS equation, this theory can be applied to the description of dissipationless shock waves in BEC.

We consider BEC confined in a disk-shaped trap with the axial frequency $\omega_{z}$ much greater than the transverse one $\omega_{x}$ $=\omega_{y}=\omega_{\perp}$. We suppose that the lower density disk-shaped BEC is confined by magnetic trap and density distribution has standard Thomas-Fermi (TF) parabolic form. Let an additional potential be applied to BEC in the central part of TF profile which leads to narrow parabolic hump in the density distribution. After the central potential is switched off, the hump starts to expand against wide lower density TF profile leading to generation of oscillations in the transition region between high and low density condensates. Let the density of atoms in the central part of BEC be of order of magnitude $n_{0}$ and satisfy the condition $n_{0} a_{s} a_{z}^{2} \ll 1$, where $a_{s}>0$ is the $s$-wave scattering length and $a_{z}=\left(\hbar / m \omega_{z}\right)^{1 / 2}$ is the amplitude of quantum oscillations in the axial trap. Then the condensate wave function $\psi$ can be factorized as $\psi=\phi(z) \Psi(x, y)$, where $\phi(z)=\pi^{-1 / 4} a_{z}^{-1 / 2} \exp \left(-z^{2} / 2 a_{z}^{2}\right)$ is the ground state wave function of axial motion, and $\Psi(x, y, t)$ satisfies the reduced $2 \mathrm{D}$ GP equation

$$
i \hbar \Psi_{t}=-\frac{\hbar^{2}}{2 m}\left(\Psi_{x x}+\Psi_{y y}\right)+V(x, y) \Psi+g_{2 D}|\Psi|^{2} \Psi,
$$

where $V(x, y)$ is the potential of a transverse trap, $g_{2 D}$ $=2 \sqrt{2 \pi} \hbar^{2} a_{s} /\left(m a_{z}\right)$ is the effective nonlinear interaction constant, and $\Psi$ is normalized to the number of atoms, $\int|\Psi|^{2} d x d y=N$. It is known that this mean field approach can be safely used for description of disk-shaped BEC with only reservation that the expression for effective constant $g_{2 D}$ 
should be changed in the asymptotic limit of extremely low gas density (see, e.g., [9], Sec. 17.4). We shall not consider here this limit and confine ourselves to standard 2D reduction (1) of the GP equation. Then the initial distribution of density is determined by the potential $V(x, y)$ and consists of wide background with a hump in its center. We assume that the background width is much greater than hump's width, so that at the initial stage of evolution we can consider an expansion of the central part against the constant background. In a similar way, at the initial stage of evolution, when the radius of the central part does not change considerably, we can neglect the curvature of axially symmetrical distribution and consider its 1D cross section. It means that we can neglect the dependence of $\Psi$ on $y$ coordinate and consider $\Psi$ as a function of $x$ and $t$ only. As a result, we arrive at 1D NLS equation with inhomogeneous initial distribution of density. To simplify the notation, we introduce dimensionless variables $t^{\prime}=\omega_{z} t / 2, x^{\prime}=x / a_{z}, u=\left(2 \sqrt{2 \pi} a_{s} a_{z} / n_{0}\right)^{1 / 2} \Psi$. Then the initial stage of evolution of the wave function profile in the $x$ axis cross section is governed by the NLS equation,

$$
i u_{t}+u_{x x}-2|u|^{2} u=0
$$

where the primes in $t^{\prime}$ and $x^{\prime}$ are omitted for convenience of the notation.

Evolution of smooth pulses before the wave breaking point can be described in the hydrodynamic approximation which can be achieved by substitution

$$
u(x, t)=\sqrt{\rho(x, t)} \exp \left(i \int^{x} v\left(x^{\prime}, t\right) d x^{\prime}\right)
$$

and separation of the real and imaginary parts. As a result we obtain the system

$$
\frac{1}{2} \rho_{t}+(\rho v)_{x}=0, \quad \frac{1}{2} v_{t}+v v_{x}+\rho_{x}=0,
$$

where we have neglected the so-called "quantum pressure" term with higher space derivatives what is correct until the density distribution has smooth enough profile. To get simple qualitative picture of the wave breaking of BEC density distribution, let us consider idealized case with a boxlike hump in the initial distribution,

$$
\rho(x, 0)= \begin{cases}\rho_{0}, & |x|>a, \\ \rho_{1}, & |x| \leqslant a .\end{cases}
$$

Although this distribution has a parameter with dimension of length - width of the hump-it does not play any role for $t$ $\leqslant a /\left(2 \sqrt{\rho_{1}}\right)$, i.e., until two waves propagating inward the hump with sound velocity $c_{1}=2 \sqrt{\rho_{1}}$ meet at $x=0$. Hence, for this initial period of evolution the solution for $x>0$ can only depend on the self-similar variable $\xi=(x-a) / t$, centered at $x=a$, and for $x<0$ on $\xi=(x+a) / t$ centered at $x=-a$. Since the picture is symmetrical, it is enough to consider only a half of the solution corresponding to $x>0$. It is easy to find that the density is given by the formulas

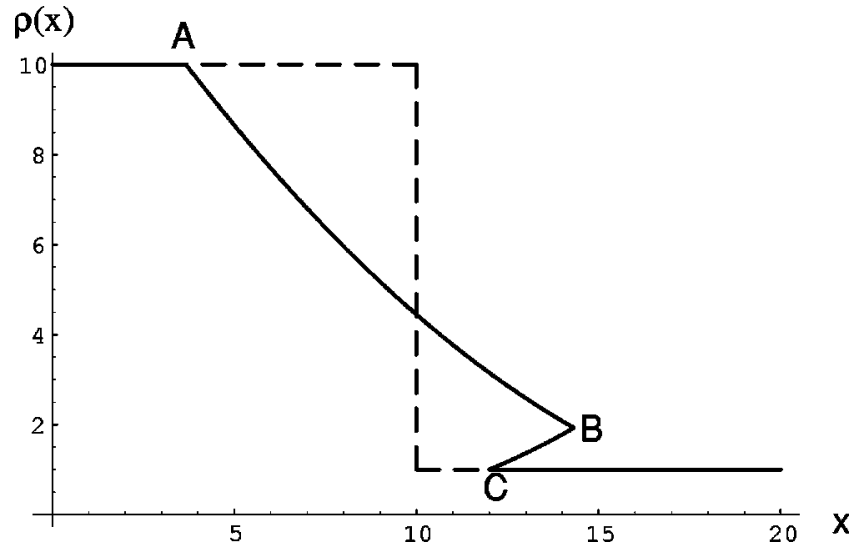

FIG. 1. Wave breaking of a boxlike initial density distribution (shown by dashed line) in the hydrodynamic approximation. The plot corresponds to the solution (6) with $a=10, \rho_{0}=1, \rho_{1}=10$, and $t=1$. Point $A$ propagates inward the box with local sound velocity $2 \sqrt{\rho_{1}}$, point $C$ propagates outward along the background density with local sound velocity $2 \sqrt{\rho_{0}}$, and point $B$ corresponds to the intersection of two simple wave solutions with profiles $A B$ and $C B$.

$$
\rho(x, t)=\left\{\begin{array}{l}
\rho_{1} \text { for } 0<x<a-2 \sqrt{\rho_{1}} t, \\
{\left[2 \sqrt{\rho_{1}}-(x-a) / 2 t\right]^{2} / 9} \\
\text { for } a-2 \sqrt{\rho_{1}} t<x<a+2\left(\sqrt{\rho_{1}}-\sqrt{\rho_{0}}\right) t, \\
{\left[2 \sqrt{\rho_{0}}+(x-a) / 2 t\right]^{2} / 9} \\
\text { for } a+2 \sqrt{\rho_{0}} t<x<a+2\left(\sqrt{\rho_{1}}-\sqrt{\rho_{0}}\right) t, \\
\rho_{0} \text { for } x>a+2 \sqrt{\rho_{0}} t
\end{array}\right.
$$

and similar formulas can be written for the velocity field $v(x, t)$. This solution describes the wave breaking phenomenon which takes place if $\rho_{1}>4 \rho_{0}$. This inequality gives an estimate for difference of densities necessary for formation of shocks. The density profile shown in Fig. 1 clearly illustrates the origin of the multivalued region which should be replaced by the oscillatory shock wave when the dispersion effects are taken into account.

For more realistic initial pulses the density profile is a smooth function without cusp points. In vicinity of the wave breaking point the solution can be approximated by a cubic function for one Riemann invariant $\lambda_{+}=v / 2+\sqrt{\rho}$ of the system (4) and by constant value for another one $\lambda_{-}=v / 2-\sqrt{\rho}$ $($ see $[12,13])$. After Galileo and scaling transformations the hydrodynamic solution can be written in the form

$$
x-\left(3 \lambda_{+}+\lambda_{-}\right) t=-\lambda_{+}^{3}, \quad \lambda_{-}=\text {const },
$$

and again for $t>0$ it has a multi-valued region of $\lambda_{+}$. It means that dispersion effects ("quantum pressure") have to be taken into account which lead to formation of dissipationless shock wave after wave breaking point.

In framework of Whitham theory of modulations $[5,14]$ one can obtain an approximate solution of the NLS equation (2) in analytic form where the dissipationless shock wave is presented as a modulated periodic nonlinear wave solution of the NLS equation. The density is expressed in terms of Jacobi elliptic function 


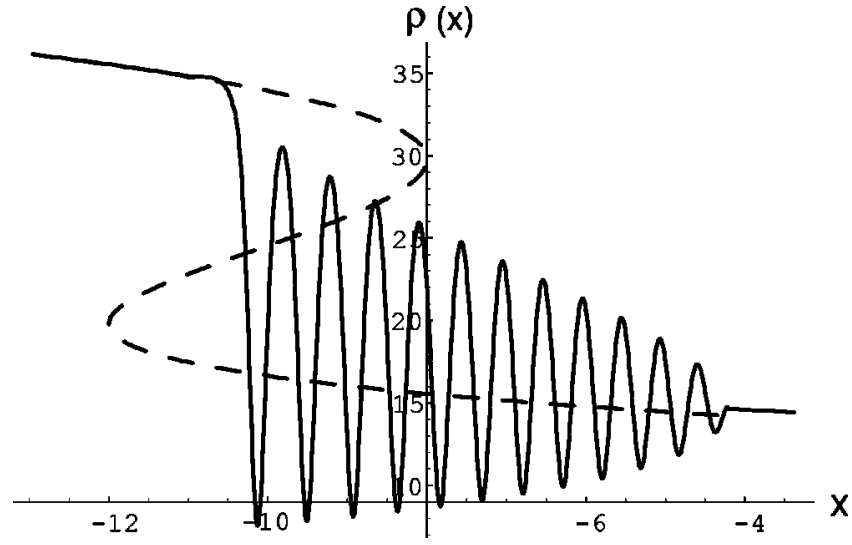

FIG. 2. Formation of dissipationless shock wave after wave breaking point according to Whitham modulation theory applied to the 1D NLS equation. The dashed line corresponds to the multivalued region arising in the hydrodynamic approximation given by Eq. (7) (it is analogous to the region between the points $B$ and $C$ in Fig. 1 ), and the solid line represents the modulated periodic wave given by Eqs. (8) and (11). Both profiles are calculated for $\bar{\lambda}=-10$ at time $t=1$.

$$
\begin{aligned}
\rho(x, t)= & |u(x, t)|^{2} \\
= & \frac{1}{4}\left(\lambda_{1}-\lambda_{2}-\lambda_{3}+\lambda_{4}\right)^{2}+\left(\lambda_{1}-\lambda_{2}\right)\left(\lambda_{3}-\lambda_{4}\right) \\
& \times \operatorname{sn}^{2}\left(\sqrt{\left(\lambda_{1}-\lambda_{3}\right)\left(\lambda_{2}-\lambda_{4}\right)} \xi, m\right),
\end{aligned}
$$

where

$$
\begin{gathered}
\xi=x-\left(\lambda_{1}+\lambda_{2}+\lambda_{3}+\lambda_{4}\right) t, \\
m=\frac{\left(\lambda_{1}-\lambda_{2}\right)\left(\lambda_{3}-\lambda_{4}\right)}{\left(\lambda_{1}-\lambda_{3}\right)\left(\lambda_{2}-\lambda_{4}\right)},
\end{gathered}
$$

and parameters $\lambda_{i}, i=1,2,3,4$, change slowly along the dissipationless shock. Their dependence on $x$ and $t$ is determined implicitly by the solution

$$
\begin{gathered}
x-v_{i}(\lambda) t=w_{i}(\lambda), \quad i=1,2,3, \\
\lambda_{4}=\bar{\lambda}=\mathrm{const}
\end{gathered}
$$

of Whitham equations, where Whitham velocities $v_{i}$ and $w_{i}$ are given by quite complicated expressions in terms of elliptic integrals (see $[12,13])$ :

$$
\begin{gathered}
w_{i}=-\frac{8}{35} w_{i}^{(3)}+\frac{4}{5} \bar{\lambda} w_{i}^{(2)}-\frac{1}{35} \bar{\lambda}^{2} v_{i}(\lambda)+\frac{1}{35} \bar{\lambda}^{3}, \\
i=1,2,3, \\
w_{i}^{(k)}=W^{(k)}+\left(v_{i}-s_{1}\right) \partial_{i} W^{(k)}, \\
W^{(1)}=V=s_{1}, \quad W^{(2)}=\frac{3}{8} s_{1}^{2}-\frac{1}{2} s_{2}, \\
W^{(3)}=\frac{5}{16} s_{1}^{3}-\frac{3}{4} s_{1} s_{2}+\frac{1}{2} s_{3},
\end{gathered}
$$
$|u(x, y)|^{2}$
(a) $t=0$

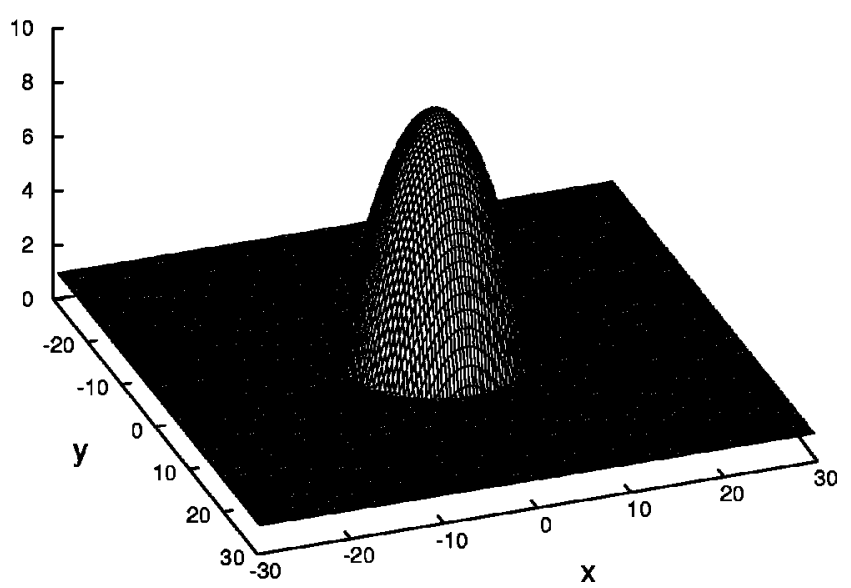

(b) $t=2$

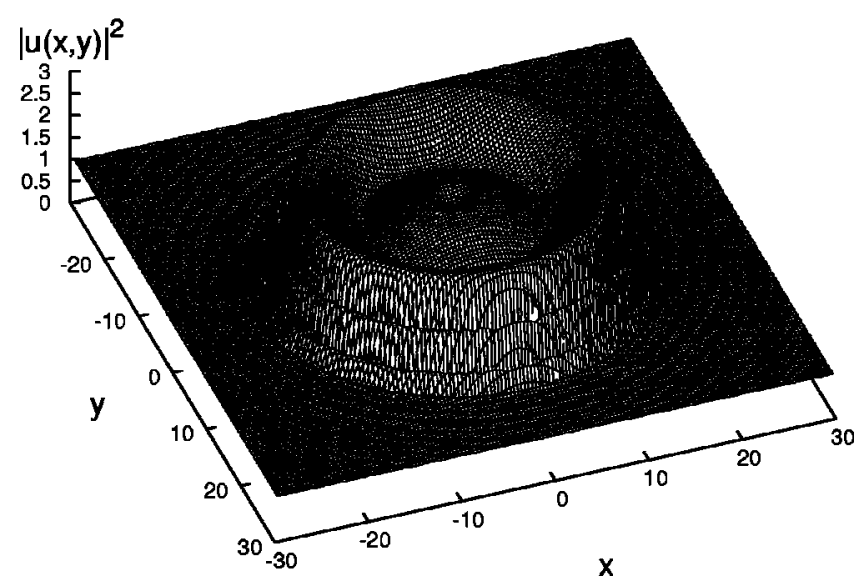

FIG. 3. (a) Two-dimensional initial distribution of BEC density with paraboloid hump on a constant background given by Eq. (19) with $a=10, \rho_{0}=1$, and $\rho_{1}=10$. (b) Two-dimensional density distribution of BEC after time $t=2$ of the evolution from the initial paraboloid density on constant background according to a numerical solution of the 2D NLS equation (18).

$$
v_{i}(\lambda)=\left(1-\frac{L}{\partial_{i} L} \partial_{i}\right) V, \quad \partial_{i} \equiv \partial / \partial \lambda_{i}, \quad i=1,2,3,4,
$$

where

$$
L=\frac{K(m)}{\sqrt{\left(\lambda_{1}-\lambda_{3}\right)\left(\lambda_{2}-\lambda_{4}\right)}}
$$

is a wavelength, $K(m)$ is the complete elliptic integral of the first kind, and $s_{1}, s_{2}, s_{3}$ are determined by the expressions

$$
s_{1}=\sum_{i} \lambda_{i}, \quad s_{2}=\sum_{i<j} \lambda_{i} \lambda_{j}, \quad s_{3}=\sum_{i<j<k} \lambda_{i} \lambda_{j} \lambda_{k} .
$$

Equations (12) can be solved with respect to $\lambda_{i}, i=1,2,3$, giving them as functions of $x$ and $t$. Subsequent substitution of these functions $\lambda_{i}(x, t), i=1,2,3$, into Eq. (8) yields the modulated periodic wave which represents the dissipationless shock wave. The resulting profile of density in dissipa- 
tionless shock wave is shown in Fig. 2. At one edge it consists of the train of dark solitons, and at another edge describes small amplitude oscillations propagating with local sound velocity into unperturbed region described by smooth solution of hydrodynamical equations. The modulated periodic wave replaces the multivalued region shown by dashed line which was obtained in hydrodynamic approximation after the wave breaking point. This multivalued region is analogous to that in Fig. 1 with account of change of variables due to Galileo and scaling transformations.

To check the described above picture of formation of dissipationless shock wave and to extend it to real 2D situation, we have solved numerically the 2D generalization of Eq. (2),

$$
i u_{t}+u_{x x}+u_{y y}-2|u|^{2} u=0,
$$

with the initial condition

$$
\rho(r, 0)=\left\{\begin{array}{l}
\rho_{0}+\left(\rho_{1}-\rho_{0}\right)\left(1-r^{2} / a^{2}\right), \quad|r| \leqslant a, \\
\rho_{0}, \quad|r|>a,
\end{array}\right.
$$

where $r=\left(x^{2}+y^{2}\right)^{1 / 2}$. Plots of two-dimensional density distribution are shown in Fig. 3 at initial moment $t=0$ and after time evolution $t=2$. As we see, the parabolic hump expands with formation of dissipationless shock wave in the transition layer between high density region and low density one. To see more clearly the evolution of the hump, its cross section $(y=0)$ profiles are shown in Fig. 4 at different values of time $t$. Slowly propagating dark solitons are clearly seen as well as small amplitude sound waves propagating into undisturbed low density region. Dissipationless shock wave generated at the right side of the profile coincides qualitatively with results of analytic theory shown in Fig. 2.

Further evolution of the hump will ultimately lead to its spreading over large area with small amplitude oscillations, that is the shock wave does not persist permanently. Rather, it is a transient phenomenon caused by different values of characteristic velocities in high density hump and low den-

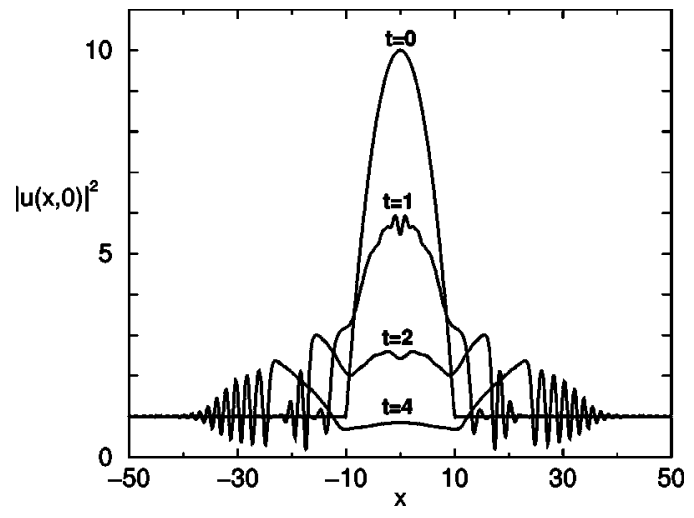

FIG. 4. Cross sections of the density profile at different evolution time according to the numerical solution of the 2D NLS equation (18) with the initial condition (19).

sity background. Slowly propagating dark solitons are generated in the transient layer between these two regions in order to reconcile two different values of velocities of disturbance propagation. This mechanism of dark soliton generation is quite general and can manifest itself in various geometries and initial BEC distributions. Formation of dark soliton trains in 1D geometry was considered in a recent paper [15]. Method of shock generation in cigar-shaped BEC by means of rapid increase of the nonlinear coupling constant using Feshbach resonance was suggested in [16].

In conclusion, we have studied analytically and numerically the process of formation of dissipationless shock waves in the density distribution of BEC. We believe that oscillations in BEC density profile observed in recent experiment [11] can be explained by this theory.

This work was supported by FAPESP (Brazil) and CNPq (Brazil). A.M.K. also thanks RFBR (Grant No. 01-0100696) for partial support.
[1] U. Ernst, A. Marte, F. Schreck, J. Schuster, and G. Rempe, Europhys. Lett. 41, 1 (1998).

[2] Y. Castin and R. Dum, Phys. Rev. Lett. 77, 5315 (1996).

[3] L. D. Landau and E. M. Lifshitz, Hydrodynamics, 4th ed. (Nauka, Moscow, 1988).

[4] A. V. Gurevich and L. P. Pitaevskii, Zh. Eksp. Teor. Fiz. 65, 590 (1973) [Sov. Phys. JETP 38, 291 (1973)].

[5] A. M. Kamchatnov, Nonlinear Periodic Waves and Their Modulations (World Scientific, Singapore, 2000).

[6] S. Burger, K. Bongs, S. Dettmer, W. Ertmer, K. Sengstock, A. Sanpera, G. V. Shlyapnikov, and M. Lewenstein, Phys. Rev. Lett. 83, 5198 (1999).

[7] J. Denschlag, J. E. Simsarian, D. L. Feder, Ch. W. Clark, L. A. Collins, J. Cubizolles, L. Deng, E. W. Hagley, K. Helmerson, W. P. Reinhardt, S. L. Rolston, B. I. Schneider, and W. D. Phillips, Science 287, 97 (2000).

[8] B. P. Anderson, P. C. Haljan, C. A. Regal, D. L. Feder, L. A. Collins, C. W. Clark, and E. A. Cornell, Phys. Rev. Lett. 86,
2926 (2001).

[9] L. Pitaevskii and S. Stringari, Bose-Einstein Condensation (Clarendon, Oxford, 2003).

[10] M. R. Andrews, D. M. Kurn, H.-J. Miesner, D. S. Durfee, C. G. Townsend, S. Inouye, and W. Ketterle, Phys. Rev. Lett. 79, 553 (1997); 80, 2967(E) (1998).

[11] E. A. Cornell, Talk at NATO Advanced Workshop "Nonlinear Waves: Classical and Quantum Aspects," Lisbon, 2003.

[12] A. M. Kamchatnov, in Nonlinearity and Disorder: Theory and Applications, edited by F. Abdullaev, O. Bang, and P. O. Sørensen (Kluwer, Boston, 2001), p. 255.

[13] A. M. Kamchatnov, R. A. Kraenkel, and B. A. Umarov, Phys. Rev. E 66, 036609 (2002).

[14] G. B. Whitham, Linear and Nonlinear Waves (Wiley, New York, 1974).

[15] B. Damski, Phys. Rev. A 69, 043610 (2004).

[16] V. M. Pérez-García, V. V. Konotop, and V. A. Brazhnyi, eprint cond-mat/0311076. 